\title{
A note on the sum of closed ideals and Riesz subspaces
}

\section{Witold Wnuk}

Received: 18 March 2014 / Accepted: 28 April 2014 / Published online: 20 May 2014 (C) The Author(s) 2014. This article is published with open access at Springerlink.com

\begin{abstract}
We indicate a broad class of closed ideals $F$ in a Banach lattice $E$ such that there exists a closed continuous (and also a closed heterogeneous) Riesz subspace $G \subset E$ satisfying the following two conditions: $G \cap F=\{0\}$ and the algebraic sum $G+F$ is not closed.
\end{abstract}

Keywords Continuous Riesz space - Discrete Riesz space - Banach lattice · Order continuous norm

Mathematics Subject Classification (2000) $\quad 46 \mathrm{~B} 42 \cdot 46 \mathrm{~A} 40 \cdot 46 \mathrm{~B} 45$

\section{Introduction}

It is well known that in every Banach space $X$ the algebraic sum $A+B$ of a closed set $A$ and a compact set $B$ is closed. The assumption of compactness is essential because $X \neq\{0\}$ always contains closed subsets whose the algebraic sum is not closed (such sets can be chosen in an arbitrary one dimensional subspace). Similar situation holds for closed linear subspaces.

$(\triangleleft)$ If $Y \subset X$ is a closed linear subspace of infinite dimension and infinite codimension, then there exists a closed linear subspace $G \subset X$ such that $Y \cap G=\{0\}$ and $Y+G \neq \overline{Y+G}$. The fact mentioned above was proved in [11] (Theorem III-14). On

This research was partially supported by The National Center of Science, Poland, Grant no. N N 201605340.

W. Wnuk $(\bowtie)$

Faculty of Mathematics and Computer Science, A. Mickiewicz University, Umultowska 87, 61-614 Poznan, Poland

e-mail:wnukwit@amu.edu.pl

Birkhäuser 
the other hand $Y+G$ is always closed whenever $Y$ is closed and $G$ is of finite dimension or $G$ is closed and finite codimensional. The question about closedness of $Y+G$ was investigated not only in the class of Banach spaces but also in more general case of Hausdorff topological vector spaces (see [4] and especially [5] where a discussion of this question and related topics are completed by many historical comments and remarks).

Our paper is also devoted to the problem of closedness of the sum $Y+G$ but we restrict considerations to Banach lattices. Our terminology and notations concerning this type of spaces (and Riesz spaces) are standard and we follow by $[1,2,10,12]$. It is natural to ask about closedness of $F+G$ for Riesz subspaces or ideals $F, G$. We should remember that the algebraic sum of two Riesz subspaces sometimes is not a Riesz subspace even if components are one dimensional. Indeed, let $E$ be a Riesz subspace in $\mathbb{R}^{\Gamma}$ containing two nonnegative functions $f, g$ such that $f\left(\gamma_{1}\right)>g\left(\gamma_{1}\right)$, $f\left(\gamma_{2}\right)<g\left(\gamma_{2}\right), f\left(\gamma_{3}\right)=g\left(\gamma_{3}\right) \neq 0$ for some $\gamma_{i} \in \Gamma, i=1,2,3$. Clearly $\mathbb{R} f$, $\mathbb{R} g$ are Riesz subspaces in $E$ while $\mathbb{R} f+\mathbb{R} g$ is not a Riesz subspace because linear independence of $f, g, f \wedge g=\inf \{f, g\}$ implies $f \wedge g \notin \mathbb{R} f+\mathbb{R} g$. On the other hand $F+G$ is a Riesz subspace whenever $F$ and $G$ are orthogonal Riesz subspaces (i.e., $|f| \wedge|g|=0$ for all $f \in F$ and all $g \in G$ ) or at least one Riesz subspace $F, G$ is an ideal. It is important and interesting that if $F_{1}, F_{2}$ are closed orthogonal Riesz subspaces or they are closed ideals in a Banach lattice then $F_{1}+F_{2}$ is closed (see [3] Theorem 5.3, [8] Theorem 1.1 or [9], [12] Proposition 1.2.2, [6] Corollary 2.3). The above statement remains true in the class of F-lattices (i.e., complete metrizable locally solid Riesz spaces) where also holds the following generalization ([6] Corollary 3.4).

Let $E$ be an F-lattice, and let $\left(I_{\gamma}\right)_{\gamma \in \Gamma}$ be a family of closed ideals in E. Define $I=\sum_{\gamma \in \Gamma} I_{\gamma}$ to be the set of all elements $z \in E$ that are of the form $z=\sum_{n=1}^{\infty} x_{n}$, where $x_{n} \in I_{\gamma_{n}}$ for every $n$, and $\left(\gamma_{n}\right)$ is a sequence in $\Gamma$. Then I is the smallest closed closed ideal in $E$ that contains all the ideals $I_{\gamma}$.

It is worth to recall that the assumption of a (topological) completeness is crucial (indeed, the authors of [16] constructed a normed lattice $E$ and a closed ideal $I \subset E$ whose algebraic sum with its orthogonal completion $I^{\mathrm{d}}$ is not closed). It occurs that a situation described in $(\triangleleft)$ is similar for closed ideals and Riesz subspaces in Banach lattices. Namely the following result was proved in [17, Theorem 2.2].

Let $I$ be a closed infinite dimensional and infinite codimensional ideal in a Banach lattice $E=(E,\|\cdot\|)$. Then there exists a closed separable discrete and $\sigma$-Dedekind complete Riesz subspace $G$ such that $I \cap G=\{0\}$ and $I+G$ is not closed.

An aim of our paper is to complete the above theorem. We will show that for many closed ideals $F$ there exist closed continuous (and closed heterogeneous) Riesz subspaces whose sums with $F$ are not closed.

\section{Auxiliary results}

Let us recall that a series $\sum_{n=1}^{\infty} x_{n}$ order converges to $x$, i.e., $x=(\mathrm{o}) \sum_{n=1}^{\infty} x_{n}$, whenever there exists a sequence $\left(y_{n}\right) \subset E$ decreasing to zero such that $\left|x-\sum_{k=1}^{n} x_{k}\right| \leqslant y_{n}$ for every $n$. If elements $x_{n}, n \in \mathbb{N}$, are positive and disjoint, then order convergence of the series $\sum_{n=1}^{\infty} x_{n}$ is equivalent to existence of $\sup _{n} x_{n}$ and there holds 
(o) $\sum_{n=1}^{\infty} x_{n}=\sup _{n} x_{n}$. Consequently considering a $\sigma$-Dedekind complete Riesz space $E$ and an order bounded sequence $\left(x_{n}\right) \subset E_{+}$of disjoint elements we obtain (o) $\sum_{n=1}^{\infty} t_{n} x_{n}=\sup _{n}\left(t_{n} x_{n}\right)^{+}-\sup _{n}\left(t_{n} x_{n}\right)^{-}$for every bounded sequence $\left(t_{n}\right) \subset \mathbb{R}$. We will say that a sequence $\left(x_{n}\right) \subset E$ is uniformly convergent to $x$, i.e., $x_{n} \stackrel{u}{\rightarrow} x$, if

$$
\exists_{u \in E_{+}} \forall_{\varepsilon>0} \exists_{N} \forall_{n>N}\left|x_{n}-x\right| \leqslant \varepsilon u .
$$

It is well known that every sequence $\left(x_{n}\right)$ norm convergent in a Banach lattice contains a subsequence uniformly convergent to $\lim _{n \rightarrow \infty} x_{n}$.

Discrete elements will be important in our further considerations. A nonzero positive element $e$ in an Archimedean Riesz space is discrete if $|x| \leqslant e$ implies $x=t e$ for some $t \in \mathbb{R}$. It is clear that two discrete elements are disjoint (=orthogonal) or they are linearly dependent. Discreteness of $e$ means exactly that the interval $[0, e]$ does not contain two nonzero disjoint elements (see [10] Theorem 26.4). Every standard basis vector is discrete in every Riesz subspace of $\mathbb{R}^{\mathbb{N}}$ containing it. Moreover $E$ is said to be discrete if every $x \in E_{+} \backslash\{0\}$ dominates a discrete element. The above definition has the following equivalent form: $E$ has a complete disjoint system $\left(e_{\gamma}\right)_{\gamma \in \Gamma}$ consisting of discrete elements, i.e., $|x| \wedge e_{\gamma}=0$ for every $\gamma$ implies $x=0$. Since every discrete element is a projection element then every positive $x$ in a discrete Riesz space $E$ has the following expression $x=\sup _{\gamma} x(\gamma) e_{\gamma}$ where the numbers $x(\gamma)$ are uniquely determined (see [1] p. 40).

On the other hand a Riesz space $E$ is called continuous if $E$ does not contain discrete elements. Therefore every nonzero positive element in a continuous Riesz space dominates a sequence whose terms are nonzero positive and pairwise disjoint (in particular nonzero ideals in $E$ are infinite dimensional). We have also the third type of a space-a Riesz space is said to be heterogeneous if it is neither discrete nor continuous. There exist many natural representatives of each class mentioned above. Sequence spaces $\ell^{p}, c_{0}$ are examples of discrete spaces, function spaces $L^{p}[0,1]$, $C[0,1]$ belong to the class of continuous Riesz spaces while products $\ell^{p} \times C[0,1]$, $c_{0} \times L^{p}[0,1]$ are heterogeneous. Let us note that every two maximal (with respect to the inclusion as a partial order) families of discrete disjoint elements in an Archimedean Riesz space $E$ have the same cardinality (more precisely, if $\left(e_{\gamma}\right)_{\gamma \in \Gamma_{1}},\left(e_{\gamma}\right)_{\gamma \in \Gamma_{2}}$ are maximal families of disjoint discrete elements, then for every $\gamma_{1} \in \Gamma_{1}$ there exists, uniquely determined, $\gamma_{2} \in \Gamma_{2}$ and $t_{\gamma_{1}}>0$ such that $e_{\gamma_{2}}=t_{\gamma_{1}} e_{\gamma_{1}}$ ). A heterogeneous Riesz space $E$ contains the discrete part $E_{D}$ and the continuous part $E_{C}$. These parts are defined as follows: $E_{D}=\left\{e_{\gamma}: \gamma \in \Gamma\right\}^{\mathrm{dd}}$ where $\left(e_{\gamma}\right)_{\gamma \in \Gamma}$ is a maximal family of discrete elements and $E_{C}=E_{D}$ d. Let us note that $E_{C}$ is infinite dimensional. Clearly $E_{D}$ is discrete and $E_{C}$ is continuous. Moreover, if $F$ is a closed heterogeneous Riesz subspace in a Banach lattice $E$, then $F_{C}, F_{D}$ are closed in $E$ because they being bands in $F$ are closed in $F$. Every Dedekind complete heterogeneous Riesz space is the sum of $E_{D}$ and $E_{C}$ but in general the equality $E=E_{D}+E_{C}$ does not hold. Indeed, consider the compact space $K=[-1,0] \cup\left\{\frac{1}{n}: n \in \mathbb{N}\right\}$ and the Banach lattice $C(K)$. Clearly $C(K)_{D}=\{f \in C(K): f([-1,0])=\{0\}\}$, and $C(K)_{C}=\left\{f \in C(K): f\left(\frac{1}{n}\right)=0\right.$ for every $\left.n\right\}$ but $f \notin C(K)_{D}+C(K)_{C}$ for a nonzero constant function $f$. 
Infinite dimensional Banach lattices always contain infinite dimensional closed discrete Riesz subspaces - it is enough to choose a sequence $\left(x_{n}\right)$ of nonzero positive disjoint elements and put $F=\overline{\operatorname{span}}\left\{x_{n}: n \in \mathbb{N}\right\}$. What about continuous Riesz subspaces? A partial answer is included in a lemma below.

Lemma 2.1 Every infinite dimensional Banach lattice E contains a continuous Riesz. subspace $E_{1}$ and a heterogeneous Riesz subspace $E_{2}$ such that both $\overline{E_{1}}$ and $\overline{E_{2}}$ are discrete and $E_{1}$ is order isomorphic to $C[0,1]$. Moreover an infinite dimensional $\sigma$ Dedekind complete Banach lattice whose norm is not order continuous contains a closed order copy of $C[0,1]$.

Proof Choose a sequence $\left(x_{n}\right) \subset E_{+}$consisting of pairwise disjoint non zero elements satisfying $\sum_{n=1}^{\infty}\left\|x_{n}\right\|<\infty$. Let $\left(q_{n}\right)$ be a sequence of all rational numbers in $[0,1]$. An operator defined by $T f=\sum_{n=1}^{\infty} f\left(q_{n}\right) x_{n}$ is an order isomorphic embedding of $C[0,1]$ into $E$.

Fix $\varepsilon>0$ and $k \in \mathbb{N}$. There exist $N>k$ and $f \in C[0,1]_{+}$such that $\sum_{n=N+1}^{\infty}\left\|x_{n}\right\|<\varepsilon, f\left(q_{k}\right)=\|f\|_{\infty}=1, f\left(\left\{q_{m}: m \in\{1, \ldots, N\} \backslash\{k\}\right\}\right)=\{0\}$. We obtain $\left\|x_{k}-T f\right\|<\varepsilon$, i.e, $x_{k} \in \overline{T(C[0,1])}$, and so $\overline{T(C[0,1])}=\overline{\operatorname{span}}\left\{x_{n}: n \in \mathbb{N}\right\}$.

It is sufficient to put $E_{1}=T(C[0,1])$ and $E_{2}=\operatorname{span}\left\{x_{2 n-1}: n \in \mathbb{N}\right\}+$ $\left\{\sum_{k=1}^{\infty} f\left(q_{k}\right) x_{2 k}: f \in C[0,1]\right\}$.

If $E$ is $\sigma$-Dedekind complete and without order continuous norm, then we can find disjoint elements $\left(x_{n}\right)$ dominated by some $x \in E_{+}$and separated from zero. Consider an operator $T: C[0,1] \rightarrow E$ given by $T f=(\mathrm{o}) \sum_{n=1}^{\infty} f\left(q_{n}\right) x_{n}$ (the sum exists by the $\sigma$-Dedekind completeness). The operator $T$ is an order isomorphism which is additionally a homeomorphism because $\inf _{n}\left\|x_{n}\right\|\|f\|_{\infty} \leqslant\|T f\| \leqslant\|x\|\|f\|_{\infty}$.

We have to explain that last statement of Lemma 2.1 is well known (see [15] Theorem 1.5).

As we see sometimes the closure of a continuous Riesz subspace is discrete. Similarly the closure does not preserve discreteness - this statement is an immediate consequence of the following result (see [15] Theorem 6.4 or [14] Theorem 4).

Theorem 2.2 For a discrete $\sigma$-Dedekind complete Banach lattice E the following are equivalent.

(a) Every closed Riesz subspace of $E$ is discrete.

(b) The closure of every discrete Riesz subspace of $E$ is discrete.

(c) The norm on $E$ is order continuous.

Therefore it may happen that the closure of a discrete Riesz subspace is heterogeneous (the closure can not be continuous because discrete elements in $F$ are also discrete in $\bar{F})$.

If $E=(E,\|\cdot\|)$ is a Banach lattice then we can associate with $E$ the ideal of elements possessing order continuous norm

$$
E_{A}=\left\{x \in E:|x| \geqslant x_{\alpha} \downarrow 0 \Rightarrow\left\|x_{\alpha}\right\| \rightarrow 0\right\} .
$$

The ideal $E_{A}$ is always closed, all discrete elements in $E$ belong to $E_{A}$, and $E_{A}$ contains all ideals $I \subset E$ with the property that the norm $\|\cdot\|$ restricted to $I$ is order continuous. 
The equality $E_{A}=\{0\}$ is possible-consider a space $C(K)$ where no point of $K$ is isolated. On the other hand $E_{A}$ can be order dense. Indeed $E_{A}=\overline{\operatorname{span}}\left\{e_{\gamma}: \gamma \in \Gamma\right\}$ whenever $\left(e_{\gamma}\right)_{\gamma \in \Gamma}$ is a complete disjoint system consisting of discrete elements in $E$. Our succeeding lemma shows that closed continuous Riesz subspaces in a discrete Banach lattice are almost disjoint with $E_{A}$.

Lemma 2.3 Let $E$ be a discrete Banach lattice. If $F \subset E$ is a closed continuous (heterogeneous) Riesz subspace, then $F \cap E_{A}=\{0\}\left(F_{C} \cap E_{A}=\{0\}\right)$.

Proof It is sufficient to consider the case when $F$ is continuous because $F_{C}$ is a closed continuous Riesz subspace for a closed heterogeneous subspace $F$.

Assume $0 \neq y \in F_{+} \cap E_{A}$. Since $F \cap E_{A}$ is a closed Riesz subspace in $E_{A}$ then according to Theorem 2.2 there exists a discrete (in $F \cap E_{A}$ ) element $e \in[0, y]$. Let us note that $F \cap E_{A}$ is an ideal in $F$, and so $e$ is a discrete element in $F$, a contradiction.

Theorem 2.2 implies that discrete Banach lattices with order continuous norm do not contain any closed continuous Riesz subspaces. Such possibility occurs also in some Banach lattices whose norm is not order continuous.

Corollary 2.4 If $E$ is a discrete Banach lattice such that $\operatorname{dim} E / E_{A}<\infty$, then every closed Riesz subspace in $E$ is discrete.

Proof Let $F \subset E$ be a closed infinite dimensional Riesz subspace and let $Q: E \rightarrow$ $E / E_{A}$ be the canonical quotient map. If $F$ were continuous or heterogeneous, then by Lemma $2.3 Q(F)$ or $Q\left(F_{C}\right)$ would be infinite dimensional subspaces in $E / E_{A}$ which is impossible.

What are examples of Banach lattices satisfying the assumption of the above corollary? The simplest one is

$$
c(\Gamma)=\left\{f: \Gamma \rightarrow \mathbb{R}: \exists_{c \in \mathbb{R}} \forall_{\varepsilon>0}\{\gamma:|f(\gamma)-c| \geqslant \varepsilon\} \text { is finite }\right\}
$$

where $c(\Gamma)$ is considered with the norm $\|f\|=\sup _{\gamma}|f(\gamma)|$. The following result is an immediate consequence of Corollary 2.4.

Corollary 2.5 For an arbitrary set $\Gamma$ every closed Riesz subspace in the Banach lattice $c(\Gamma)$ is discrete.

The proofs of our main results use a procedure which allows to join some Riesz subspaces. The procedure is described in next lemma where we will use the notation $X \perp Y$ that means the orthogonality (= disjointness) of sets $X, Y$, i.e., $|x| \wedge|y|=0$ for every $x \in X$ and $y \in Y$.

Lemma 2.6 Let $E$ be a Banach lattice and suppose that $F_{k} \subset E, k \in \mathbb{N}$, are closed Riesz subspaces satisfying the condition $F_{m} \perp F_{j}$ for distinct $m, j$. Then the order sum of $F_{k}$ 's, i.e., the space $F=(0)\left(\oplus F_{k}\right)=\left\{x \in E: x=(0) \sum_{k=1}^{\infty} x(k)\right.$ where $x(k) \in$ $\left.F_{k}\right\}$ is a closed Riesz subspace. 
Proof It is easy to check that $F$ is a Riesz subspace. Suppose $F_{+} \ni x_{n}=$ (o) $\sum_{k=1}^{\infty} x_{n}(k) \underset{n \rightarrow \infty}{\longrightarrow} x$. Clearly $x \in E_{+}$and $x_{n}(k)=\left|x_{n}(k)\right|$. The inequality $\left|x_{n}(k)-x_{m}(k)\right| \leqslant\left|x_{n}-x_{m}\right|$ shows that $\left(x_{n}(k)\right)_{n=1}^{\infty}$ is a Cauchy sequence for every $k$. If $x(k)=\lim _{n \rightarrow \infty} x_{n}(k)$, then $x(k) \in F_{k}$. Let $P_{j}$ the band projection mapping the Dedekind completion $E^{\delta}$ of $E$ onto the band in $E^{\delta}$ generated by $F_{j}$. Since $E$ is norm complete then for every $j$ we can find a subsequence $\left(n_{m}\right)$ such that $x_{n_{m}} \stackrel{u}{\rightarrow} x$ and $x_{n_{m}}(j) \stackrel{u}{\rightarrow} x(j)$. Therefore $x_{n_{m}}(j)=P_{j} x_{n_{m}} \stackrel{u}{\rightarrow} P_{j} x$, and so $x(j)=P_{j} x \leqslant x$. Suppose that $y \in E$ is such that $x(j) \leqslant y$ for all $j \in \mathbb{N}$. There holds $x_{n}(j) \leqslant x_{n}(j)-x(j)+y=P_{j}\left(x_{n}-x\right)+y \leqslant P_{j}\left(\left|x_{n}-x\right|\right)+y \leqslant\left|x_{n}-x\right|+y$. Hence $x_{n}=(\mathrm{o}) \sum_{j=1}^{\infty} x_{n}(j)=\sup _{j} x_{n}(j) \leqslant\left|x_{n}-x\right|+y$ and finally $x \leqslant y$. We have just proved $x=\sup _{j} x(j)=(\mathrm{o}) \sum_{j=1}^{\infty} x(j) \in F$.

Consider a sequence $\left(x_{n}\right) \subset F$ converging to $x$. Since $F \ni x_{n}^{+} \rightarrow x^{+}$and $F \ni$ $x_{n}^{-} \rightarrow x_{n}^{-}$, then $x^{+}, x^{-} \in F$. Therefore $x=x^{+}-x^{-} \in F$, i.e., $F=\bar{F}$.

We will also need a condition implying that the algebraic sum $H+F$ of closed subspaces $H, F$ is not closed. Such condition was formulated, not in an explicit form, in [13] (Section 6). Since it is used twice in proofs of our main results, and for the sake of completeness, we recall the condition.

Lemma 2.7 Let $H, F$ be closed linear subspaces in a Banach space $X$. If $H \cap F=\{0\}$ and there exist sequences $\left(x_{n}\right) \subset X,\left(y_{n}\right) \subset F$ such that $\left\|x_{n}\right\| \rightarrow 0, \inf _{n}\left\|y_{n}\right\|>0$ and $x_{n}+y_{n} \in H$, then $H+F$ is not closed.

Proof The closedness of $H$ and $F$ implies that the natural projection $P: H+F \rightarrow F$ has the closed graph. Therefore if $H+F$ were closed, then $P$ would be continuous by the closed graph theorem, but $\left\|\left(x_{n}+y_{n}\right)-y_{n}\right\| \rightarrow 0$ while $\left\|P\left(\left(x_{n}+y_{n}\right)-y_{n}\right)\right\|=$ $\left\|P y_{n}\right\| \nrightarrow 0$, a contradiction.

Moreover we will apply the following result showed in [17] (p. 437, the proof of Lemma 2.1).

Lemma 2.8 Let I be a closed ideal in a Banach lattice E. If $Q: E \rightarrow E / I$ is the canonical quotient map and a sequence $\left(Q\left(y_{n}\right)\right)_{n=1}^{\infty}$ consists of nonzero positive disjoint elements, then there exist disjoint elements $x_{n} \in E_{+} \backslash I, n=1,2, \ldots$, such that $Q\left(x_{n}\right)=Q\left(y_{n}\right)$ for each $n$.

\section{Main results}

Theorems presented in this section concern two types of (closed) ideals $F$-either the quotient norm in $E / F$ is not order continuous or $F$ is contained in a Banach lattice with order continuous norm. Theorem 2.2 shows a class of Banach lattices where closed continuous and closed heterogeneous Riesz subspaces do not exist. Therefore if we want to prove that for a closed ideal $F \subset E$ there is a closed (heterogeneous) Riesz subspace $G$ in $E$ with $F+G \neq \overline{F+G}$ we have to assume something about $E$ or $F$. 
Theorem 3.1 Let $E$ be a $\sigma$-Dedekind complete Banach lattice and let $F \subset E$ be a closed infinite dimensional ideal such that the quotient norm on $E / F$ is not order continuous. There exists a closed continuous Riesz subspace $G_{1} \subset E$ and a closed heterogeneous Riesz subspace $G_{2} \subset E$ such that $F \cap G_{i}=\{0\}$ and $F+G_{i}$ is not closed for $i=1,2$.

Remark 3.2 If $E$ is a $\sigma$-Dedekind complete Banach lattice and $E \neq E_{A}$, then the quotient norm on $E / E_{A}$ is not order continuous (see [16] Theorem 3.3), and so Theorem 3.1 can be applied for $F=E_{A}$ whenever $\operatorname{dim} E_{A}=\infty$.

Proof The quotient $E / F$ contains a separated from zero sequence $\left(Q\left(y_{n}\right)\right)$ of pairwise disjoint elements included in some interval $[0, Q(y)]$ where $Q: E \rightarrow E / F$ is the canonical quotient map (see [2] Theorem 4.14 or [12] Theorem 2.4.2). Applying Lemma 2.8 we find pairwise disjoint $x_{n}^{\prime} \in E_{+} \backslash F$ such that $Q\left(x_{n}^{\prime}\right)=Q\left(y_{n}\right)$. Therefore the elements $x_{n}=x_{n}^{\prime} \wedge|y|$ are pairwise disjoint, order bounded and separated from zero because $0<\inf _{n}\left\|Q\left(y_{n}\right)\right\|=\inf _{n}\left\|Q\left(x_{n}\right)\right\| \leqslant \inf _{n}\left\|x_{n}\right\|$. Moreover $x_{n} \notin F$. Let

$-\mathcal{N}_{1}=\left\{n: x_{n} \in F^{\mathrm{dd}}\right\}$,

$-\mathcal{N}_{2}=\left\{n: x_{n} \in F^{\mathrm{d}}\right\}$,

$-\mathcal{N}_{3}=\left\{n: x_{n} \notin F^{\mathrm{dd}} \cup F^{\mathrm{d}}\right\}$.

The family $\left\{\mathcal{N}_{i}: i=1,2,3\right\}$ forms a partition of $\mathbb{N}$. Hence at least one set $\mathcal{N}_{j}$ is infinite. Since $F+F^{\mathrm{d}}$ is order dense in $E$ (see [1] Theorem 1.25), then for the ideal $\mathcal{J}\left(x_{n}\right)$ in $E$ generated by $\left\{x_{n}\right\}$ we obtain $\mathcal{J}\left(x_{n}\right) \cap F_{+} \neq\{0\}$ for $n \in \mathcal{N}_{1} \cup \mathcal{N}_{3}$. Fix $j \in\{1,2,3\}$ such that $\mathcal{N}_{j}$ is infinite and choose norm one elements $h_{n}$ with $n \in \mathcal{N}_{j}$ satisfying:

$-h_{n} \in \mathcal{J}\left(x_{n}\right) \cap F_{+}$if $j=1$ or $j=3$,

$-h_{n} \in F_{+}$and $h_{k} \wedge h_{m}$ for distinct $k, m \in \mathcal{N}_{j}$ if $j=2$,

Let infinite sets $N_{i}=\left\{n_{1}^{i}, n_{2}^{i}, \ldots\right\}, i=1,2, \ldots$, form a partition of $\mathcal{N}_{j}$. Putting $\mathcal{F}_{i}=\left\{x_{n_{1}^{i}}+h_{n_{1}^{i}}, x_{n_{2}^{i}}, x_{n_{3}^{i}}, \ldots\right\}$ we obtain $\mathcal{F}_{i} \perp \mathcal{F}_{k}$ for $i \neq k$ and $a \wedge b=0$ for arbitrary $a, b \in \mathcal{F}_{i}$.

Consider an operator $T: C[0,1] \rightarrow E$ defined by

$$
T_{i} f=f\left(q_{1}\right)\left(\frac{1}{i} x_{n_{1}^{i}}+h_{n_{1}^{i}}\right)+(\mathrm{o}) \sum_{k=2}^{\infty} \frac{1}{i} f\left(q_{k}\right) x_{n_{k}^{i}}
$$

where $\left(q_{n}\right)$ is a sequence of all rational numbers in $[0,1]$. The operator $T_{i}$ is well defined by the $\sigma$-Dedekind completeness of $E$ and it is an order isomorphism being at the same time a homeomorphism because

$$
\frac{1}{i} \inf _{n}\left\|x_{n}\right\|\|f\|_{\infty} \leqslant\left\|T_{i} f\right\| \leqslant\|f\|_{\infty}\left\|x^{(i)}\right\|,
$$

where $x^{(i)}=\sup \mathcal{F}_{i}$. Hence $G_{i}=T_{i}(C[0,1])$ are closed continuous Riesz subspaces in $E$ and $G_{i} \perp G_{j}$. The space $G=(\mathrm{o})\left(\oplus G_{n}\right)$ is also a continuous closed Riesz subspace and $F \cap G=\{0\}$. Indeed, if $0<x \in G$, then $x \geqslant T_{i} f \supsetneqq 0$ for some $i$ 
and $f \supsetneqq 0$. Since $\left(q_{n}\right)_{n} \geqslant M$ is dense in $[0,1]$ for every $M \in \mathbb{N}$, then $f\left(q_{k}\right)>0$ for infinitely many $k$ 's. Hence $x \geqslant \frac{1}{i} f\left(q_{k}\right) x_{n_{k}^{i}} \neq 0$ for many $k \geqslant 2$, and so $x \notin F$ because $F$ is an ideal. Put $z_{i}=\sup _{k} x_{n_{k}^{i}}$ and $x=\sup _{n} x_{n}$. We obtain $\left\|\frac{1}{i} z_{i}\right\| \leqslant \frac{1}{i}\|x\| \rightarrow 0$ and $\frac{1}{i} z_{i}+h_{n_{1}^{i}}=T_{i}(1) \in G$. Hence sequences $\left(\frac{1}{i} z_{i}\right)$ and $\left(h_{n_{1}^{i}}\right)$ have properties assumed in Lemma 2.7, i.e., $G+F$ is not closed.

Define $G_{1}{ }^{\prime}=\overline{\operatorname{span}}\left\{x_{n_{2}^{1}}, x_{n_{3}^{1}}, \ldots\right\}$. Clearly $G_{1}{ }^{\prime}$ is a closed discrete Riesz subspace and $G_{1}{ }^{\prime} \cap F=\{0\}$. Indeed, every $x \in G_{1}{ }^{\prime} \backslash\{0\}$ is of the form $x=\sum_{i=2}^{\infty} t_{i} x_{n_{i}}$ and at least one $t_{i}$ is nonzero. For $t_{i} \neq 0$ we obtain $|x| \geqslant\left|t_{i}\right| x_{n_{i}^{1}} \notin F$, and so $x \notin F$ because $F$ is an ideal. Let $G^{\prime}=(\mathrm{o})\left(\oplus G_{n}{ }^{\prime}\right)$ where $G_{n}{ }^{\prime}=G_{n}$ for $n>1$. The space $G^{\prime}$ is a closed heterogeneous Riesz subspace possessing the required properties: $G^{\prime} \cap F=\{0\}$ and $G^{\prime}+F \neq \overline{G^{\prime}+F}$.

The proof of the previous result started with an observation that there exists a sequence separated from zero which terms are pairwise disjoint order bounded and lying outside $F$. Such sequence allowed us to construct an order and topological copies of the continuous Banach lattice $C[0,1]$. The same idea can not be repeated when $E$ has order continuous norm because the order continuity of a norm means exactly that order bounded sequences of disjoint elements are norm null (see [15] Theorem 1.1 or [2] Theorem 4.14 or [12] Theorem 2.4.2). Moreover Banach lattices with order continuous norms do not contain any closed Riesz subspace order isomorphic to $C[0,1]$ (see [15] Theorem 1.5 or [2] Theorem 4.56 and Example 4.55 or [12] Corollary 5.16 and remarks preceding it). Therefore, if we want to prove a theorem analogous to Theorem 3.1 covering the case of Banach lattices with order continuous norm, we need another method. It is well known that a closed ideal in a Banach lattice $E$ with order continuous norm is a band. Moreover if $E$ is continuous, then every non trivial band $\mathrm{F}$ (i.e., $\{0\} \neq F \neq E$ ) is infinite dimensional and its codimension is infinite too (because finite dimensional ideals are discrete).

Theorem 3.3 Let E be a continuous Banach lattice with order continuous norm. If $F \subset E$ is a non trivial band, then there exists a continuous closed Riesz subspace $H_{1} \subset E$ and a closed heterogeneous Riesz subspace $H_{2} \subset E$ such that $F \cap H_{i}=\{0\}$ and $F+H_{i}$ is not closed for $i=1,2$.

Proof Suppose first that $E$ has a weak order unit. According to a Representation Theorem (see [15] Theorem 1.10 or [7] Theorem 1.b.14) there exists a finite atomless measure space $(S, \Sigma, \mu)$ such that $E$ can be identified (by an order isomorphism) with an ideal $I$ such that $L^{\infty}(\mu) \subset I \subset L^{1}(\mu)$. Since simple functions are order dense in $L^{1}(\mu)$ we can choose disjoint sets $A, C \in \Sigma$ with a positive measure which characteristic functions $1_{A}, 1_{C}$ belong to $F^{\mathrm{d}}$ (= the orthogonal complement of $F$ in $E$ ) and $F$ respectively. By the non-atomicity of $\mu$ we find sets $A_{n}^{k}, C_{n}^{k} \in \Sigma, n \in \mathbb{N}$, $k \in\left\{1,2, \ldots, 2^{n}\right\}$ having the following properties:

(a) $A=A_{1}^{1} \cup A_{1}^{2}, C=C_{1}^{1} \cup C_{1}^{2}$,

(b) $A_{n}^{k} \cap A_{n}^{j}=\emptyset=C_{n}^{k} \cap C_{n}^{j}$ for every $n$ and distinct $k, j \in\left\{1,2, \ldots, 2^{n}\right\}$,

(c) $A_{n}^{k}=A_{n+1}^{2 k-1} \cup A_{n+1}^{2 k}, C_{n}^{k}=C_{n+1}^{2 k-1} \cup C_{n+1}^{2 k}$,

(d) $\mu\left(A_{n}^{k}\right)=2^{-n} \mu(A), \mu\left(C_{n}^{k}\right)=2^{-n} \mu(C)$. 
Choose $s, t>0$ and put

(+) $\quad G=\operatorname{span}\left(\left\{t 1_{A_{n}^{k}}+s 1_{C_{n}^{k}}: n \in \mathbb{N}, k \in\left\{1,2, \ldots, 2^{n}\right\}\right\} \cup\left\{t 1_{A}+s 1_{C}\right\}\right)$, where $1_{B}$ is the characteristic function of the set $B$.

The space $G$ is a continuous Riesz space because every $g \in G$ is of the form

(*) $\quad g=\sum_{k=1}^{2^{n}} t_{k}\left(t 1_{A_{n}^{k}}+s 1_{C_{n}^{k}}\right)$ for some $n$.

We claim $\bar{G} \cap F=\{0\}$ and $\bar{G}$ is continuous. The $L^{1}$-norm $\|\cdot\|_{1}$ restricted to $E$ is weaker than the original norm of $E$ (see [2] Theorem 4.3), and so $\bar{G} \subset \bar{G}^{1} \cap E$ where $\bar{G}^{1}$ denotes the closure in the $L^{1}$-norm topology. Suppose $0 \neq h \in \bar{G}^{1} \cap F$. There exists $g \in G$ satisfying $\|h-g\|_{1}<\varepsilon$ where $0<\varepsilon<\|h\|_{1} \frac{t \mu(A)}{t \mu(A)+s \mu(C)}$. Let $P: E \rightarrow F$ be the band projection. We obtain $\max \left(\|h-P g\|_{1},\|(\mathrm{Id}-P) g\|_{1}\right) \leqslant\|h-g\|_{1}<\varepsilon$ because $|h-P g| \wedge|(\mathrm{Id}-P) g|=0$. Since $g$ is of the form (*) we can express the above inequalities in the following way

$$
\begin{aligned}
\varepsilon>\|(\mathrm{Id}-P) g\|_{1} & =t \sum_{j=1}^{2^{n}}\left|t_{j}\right| 2^{-n} \mu(A) \text { and } \varepsilon>\|h-P g\|_{1} \\
& \geqslant\|h\|_{1}-s \sum_{j=1}^{2^{n}}\left|t_{j}\right| 2^{-n} \mu(C) .
\end{aligned}
$$

Elementary calculations show that

$$
\frac{2^{n} \varepsilon}{t \mu(A)}>\sum_{j=1}^{2^{n}}\left|t_{j}\right| \geqslant\left(\|h\|_{1}-\varepsilon\right) \frac{2^{n}}{s \mu(C)}
$$

i.e., $\varepsilon \geqslant\|h\|_{1} \frac{t \mu(A)}{t \mu(A)+s \mu(C)}$, a contradiction. Hence $\bar{G} \cap F \subset \bar{G}^{1} \cap F=\{0\}$.

Every set $B \in \Sigma$ determines a positive (and so continuous) operator $T_{B}: E \rightarrow E$ defined by $T_{B} f=f 1_{B}\left(T_{B}\right.$ is well defined because $E$ is an ideal in $\left.L^{1}(\mu)\right)$.

Since for every function $g \in G$ there holds $T_{A \cup C} g=g$ and $\left(T_{A_{n}^{j}}+T_{C_{n}^{j}}\right) g \in G$, then $h=h 1_{A \cup C}\left(\mu\right.$-almost everywhere) and $\left(T_{A_{n}^{j}}+T_{C_{n}^{j}}\right) h \in \bar{G}$ whenever $h \in \bar{G}$. Fix a positive non zero function $h \in \bar{G}$. There exists $n \in \mathbb{N}$ such that the cardinality of the set $\mathcal{H}=\left\{j \in\left\{1, \ldots, 2^{n}\right\}: \mu\left(\operatorname{supp} h \cap\left(A_{n}^{j} \cup C_{n}^{j}\right)\right) \neq 0\right\}$ is at least two. Indeed, in the contrary case for every $m$ we can find at most one $j_{m} \in\left\{1, \ldots, 2^{m}\right\}$ with $\mu\left(\operatorname{supp} h \cap\left(A_{m}^{j_{m}} \cup C_{m}^{j_{m}}\right)\right)>0$ which is impossible because

$$
\begin{aligned}
0<\mu(\operatorname{supp} h) & =\sum_{k=1}^{2^{m}} \mu\left(\operatorname{supp} h \cap\left(A_{m}^{k} \cup C_{m}^{k}\right)\right)=\mu\left(\operatorname{supp} h \cap\left(A_{m}^{j_{m}} \cup C_{m}^{j_{m}}\right)\right) \\
& \leqslant \frac{1}{2^{m}}(\mu(A)+\mu(C)),
\end{aligned}
$$

i.e., $\mu(\operatorname{supp} h)=0$, and so $h=0$. We have just shown $\bar{G}$ is continuous: functions $h 1_{A_{n}^{j} \cup C_{n}^{j}}$, for $j \in \mathcal{H}$, are disjoint non zero functions in $\bar{G}$ majorized by $h$. 
Remembering that simple functions are order dense in $L^{1}(\mu), E$ is an ideal in $L^{1}(\mu)$, $\operatorname{dim} F^{\mathrm{d}}=\infty, \operatorname{dim} F=\infty$, we are able to find disjoint sets $A_{n}$ and disjoint sets $C_{n}$, $n \in \mathbb{N}$, and positive numbers $t_{n}, s_{n}$ with the following properties $1_{A_{n}} \in F^{\mathrm{d}} \backslash\{0\}$, $1_{C_{n}} \in F,\left\|t_{n} 1_{A_{n}}\right\| \rightarrow 0,\left\|s_{n} 1_{C_{n}}\right\|=1$. It is clear that $\mu\left(A_{m} \cap C_{j}\right)=0$ for all $m, j$. Let $G_{n}$ be a Riesz subspace determined by sets $A_{n}, C_{n}$ and numbers $t_{n}, s_{n}$ in the same way as the Riesz subspace $G$ considered above (see $(+))$. Put $H=(\mathrm{o})\left(\oplus \overline{G_{n}}\right)$. The Riesz subspace $H$ is continuous, closed, and $H \cap F=\{0\}$. It is obvious that $t_{n} 1_{A_{n}}+s_{n} 1_{C_{n}} \in H$. Hence sequences $\left(t_{n} 1_{A_{n}}\right),\left(s_{n} 1_{C_{n}}\right)$ have properties assumed in Lemma 2.7, i.e., $H+F$ is not closed.

Modifying the Riesz subspace $\overline{G_{1}}$ we can easy construct a heterogeneous closed Riesz subspace satisfying the required conditions. Since $\mu$ is atomless we are able to find disjoint sets $B_{k} \subset A_{1}, k \in \mathbb{N}$, with positive measure. Let $G_{1}^{\prime}=\overline{\operatorname{span}}\left\{1_{B_{k}}: k \in \mathbb{N}\right\}$ and $G_{n}{ }^{\prime}=\overline{G_{n}}$ for $n>1$. The Riesz subspace $H^{\prime}=(\mathrm{o})\left(\oplus G_{n}^{\prime}\right)$ is closed and heterogeneous. Moreover $H^{\prime} \cap F=\{0\}$ and $H^{\prime}+F \neq \overline{H^{\prime}+F}$.

Suppose now that $E$ has no weak units. Choose $0<x \in F$ and $0<y \in F^{\mathrm{d}}$. Denote by $P$ the band projection onto $\{x+y\}^{\mathrm{dd}}$. The inclusion $\{x\}^{\mathrm{dd}} \subset P F$ and the equality $\{y\}^{\mathrm{dd}} \cap P F=\{0\}$ show the band $P F$ is a non trivial band in $P E$ where $x+y$ is a weak unit. By the first part of our proof there exists a continuous (heterogeneous) closed Riesz subspace $H \subset P E$ such that $H \cap P F=\{0\}$ and $H+P F \neq \overline{H+P F}$. Let us note that if $x \in H \cap F$, then $x \in H \subset P E$, and so $x=P x \in P H \cap P F=H \cap P F=\{0\}$.

Choose a sequence $\left(h_{n}+P f_{n}\right) \subset H+P F$ convergent to $x \notin H+P F$. If $H+F$ were closed, then it would be $x=h+f \in H+F$ but $h_{n}+P f_{n}=P\left(h_{n}+P f_{n}\right) \rightarrow$ $P x=h+P f$, i.e., $x=h+P f \in H+P F$, a contradiction.

Acknowledgments I am very grateful to the referee for his comments and suggestions concerning the first version of this paper.

Open Access This article is distributed under the terms of the Creative Commons Attribution License which permits any use, distribution, and reproduction in any medium, provided the original author(s) and the source are credited.

\section{References}

1. Aliprantis, C., Burkinshaw, O.: Locally Solid Riesz Spaces with Applications to Economics, Mathematical Surveys and Monographs, 2nd edn, vol 105. American Mathematical Society, Providence, RI (2003)

2. Aliprantis, C., Burkinshaw, O.: Positive Operators. Springer, Berlin (2006)

3. Davis, E.B.: The structure and ideal theory of the predual of a Banach lattice. Trans. Am. Math. Soc. 131, 544-555 (1968)

4. Drewnowski, L.: On minimal topological linear spaces and strictly singular operators. Comment. Math. Tomus Specialis II, 89-106 (1979)

5. Drewnowski, L.: Quasi-complements in F-spaces. Studia Math. 77, 373-391 (1984)

6. Drewnowski, L.: On infinite sums of closed ideals in F-lattices. Func. Approx. Comment. Math. 44(2), 279-284 (2011)

7. Lindenstrauss, J., Tzafriri, L.: Classical Banach Spaces II. Function Spaces. Springer, Berlin, New York (1979)

8. Lotz, H.P.: Über das Spektrum positiver Operatoren. Math. Z. 108, 15-32 (1968)

9. Lotz, H.P.: A note on the sum of two closed lattice ideals. Proc. Am. Math. Soc. 44(2), 389-390 (1974)

10. Luxemburg, W.A.J., Zaanen, A.C.: Riesz Spaces I. North-Holland, Amsterdam (1971) 
11. Mackey, G.W.: On infinite dimensional linear spaces. Trans. Am. Math. Soc. 57, 155-207 (1945)

12. Meyer-Nieberg, P.: Banach Lattices. Springer, Berlin Heidelberg New York (1991)

13. Wilansky, A.: Semi-Fredholm maps of FK-spaces. Math. Z. 144, 9-12 (1975)

14. Wnuk, W.: When is the closure of an atomic Riesz subspace atomic? Bull. Acad. Pol. Sci. Math. 34, 659-694 (1986)

15. Wnuk, W.: Banach Lattices with Order Continuous Norms, Advanced Topics in Mathematics. Polish Scientific Publishers PWN, Warsaw (1999)

16. Wnuk, W., Wiatrowski, B.: On the Levi and Lebesgue properties. Indag. Math. (N.S.) 18(4), 641-650 (2007)

17. Wnuk, W.: Some remarks on the algebraic sum of ideals and Riesz subspaces. Canad. Math. Bull. 56(2), 434-441 (2013). doi:10.4153/CMB-2011-151-0 